\title{
THEORETIC INTERPLAY BETWEEN ABSTRACTION, RESOLUTION, AND FIDELITY IN MODEL INFORMATION
}

\author{
Il-Chul Moon \\ Jeong Hee Hong \\ Dept. of Industrial and Systems Engineering \\ KAIST \\ 291 Daehak-ro, Daejeon, 305-701, KOREA
}

\begin{abstract}
Modeling and simulating a real world scenario is fundamentally an abstraction that takes only part of the given scenario into the model. Furthermore, the level of detail in the model, a.k.a. the resolution, plays an important role in the modeling and simulation process. Finally, the abstraction and resolution of the model determine the fidelity of the modeling and simulation, which becomes the ultimate utility for the model users. While abstraction, resolution and fidelity are the corner stones of the modeling and simulation discipline, they are often casually utilized. Moreover, their interplay is not investigated in-depth with explicit operationalization of the concepts. This article operationalizes the concept of abstraction, resolution, and fidelity by focusing on the aspect of model information. This theoretic investigation answers propositions involving these concepts, i.e. whether or not a higher resolution model has higher fidelity and why, through set theoretic approaches.
\end{abstract}

\section{INTRODUCTION}

Modeling and simulation (M\&S) is ultimately an abstracted version of the real world. If we intend to regenerate scenarios of interest in the real world without any loss of detail, modeling is impossible (Davis and Bigelow 1998). Even if we consider only a facet of the real world, there are uncountable details that we have to take into consideration. For instance, the famous butterfly effect claims that a distant and small effect may cause a significant and large outcome after complex interactions between real world entities. Assuming that this argument is true, simulation modelers might not have the full information that is necessary to replicate the real world perfectly.

If simulation modelers admit that a simulation is an abstraction (Giunehiglia and Walsh 1992) of the real world, the M\&S community needs to engage in conceptual, theoretic, and practical discussions on abstraction because abstraction is the one mechanism that enables our community to do its work. If simulation modelers have common ground in defining the concept of abstraction, that would be a good place to begin discussions on what good or bad abstraction is, how to abstract the real world better, and how to improve current modeling practices in the M\&S community. Recent M\&S research contributions includes numerous case study reports that cannot be validated for various reasons, such as unavailable datasets, unseen futures, etc. Without numerical validation, which is inadequate in a number of cases, how can simulation modelers who have abstracted the real world be evaluated by a certain quality standard? A clear definition and theoretic investigation on abstraction would be one way to build such a standard.

Further, abstraction is closely related to other important mechanisms in M\&S. First, "resolution" (Davis and Bigelow 1998) is often used interchangeably with "abstraction". The resolution is important because the resolution of a simulation model determines the level of details in the model and because the level of details determines what to include and how to implement the model. Second, abstraction and resolution are the major determinants of the fidelity of simulation models (Roza 2004). Conceptually, less abstraction and higher resolution provides higher fidelity in the simulation models. 


\section{Moon and Hong}

Most simulation modelers are aware of the close relation between abstraction, resolution, and fidelity, but only a few simulation modelers clearly distinguish them in their academic contributions (Davis and Bigelow 1998; Roza 2004; Yilmaz et al. 2007; Mitchell and Yilmaz 2008; Turnitsa and Tolk 2008; Davis and Tolk 2007; Gross and Freeman 1997; Gross, Freeman, and William 1998; Gross and Freeman 1999). In some occasions, these concepts are casually used in research papers, so non-experts might get incorrect impressions from the papers. Moreover, even $M \& S$ experts have slightly different views on these concepts when they articulate each concept precisely. Therefore, investigating these concepts theoretically to provide a concrete grounding is interesting and critical simultaneously.

This article proposes a potential grounding for abstraction, resolution, and fidelity through mathematical and logical arguments. Given the vast amount of M\&S literature and their own views on these concepts, it should be noted that this article is just a single position in defining and theorizing the concepts. We develop theoretic arguments on abstraction, resolution, and fidelity from a simple black box model and its represented modeling features. The development mainly depends on the amount of information that the model holds and how it relates to the real world scenario that is the target of the modeling.

\section{LITERATURE REVIEW}

Before we start the theoretic discussion, we discuss the existing definitions and discussions of abstraction, resolution, and fidelity in the M\&S community and its neighbor communities, such as computer science.

\subsection{Definitions and Preliminaries of Abstraction, Resolution, and Fidelity in Model Information}

Many works (Davis and Bigelow 1998; Roza 2004; Yilmaz et al. 2007; Mitchell and Yilmaz 2008; Turnitsa and Tolk 2008; Davis and Tolk 2007; Gross and Freeman 1997; Gross, Freeman, and William 1998; Gross and Freeman 1999) have been published to define and examine the concepts and their casual definitions. For example, Davis (2000) has published numerous papers on multi-resolution modeling, and the papers had discussions and definitions of the concepts. Yilmaz et al. (Yilmaz et al. 2007; Mitchell and Yilmaz 2008) have expressed their views on multi-modeling, and their views categorize and specify multi-abstraction and multi-resolution, etc. Turnitsa and Tolk (2008); and Davis and Tolk (2007) discussed multi-modeling from the viewpoint of model interoperability and ontologies. This short list shows that many prominent M\&S researchers expressed their own views on this subject. Gross et al. (Gross and Freeman 1997; Gross, Freeman, and William 1998; Gross and Freeman 1999) have conducted research to define, measure, and evaluate fidelity. Moreover, contributions have been published in relatively recent years, i.e. 2007, and given that these concepts are the backbone of the M\&S community, it is interesting that there are still theoretic developments in this subject.

We identified a number of definitions of resolution, but the M\&S community lacks an operationalized definition of abstraction. For instance, if one of the oldest contributions regarding, the section titled "Definitions and Technical Background" in Davis and Bigelow (1998) begins with "All models are abstractions of reality..." This statement leads to a definition of resolution, but Bigelow and Davis treat the concept of abstraction as already given. This trend is similar to other M\&S papers. In the computer science discipline, abstraction is defined as follows.

- Abstraction: An abstraction, written $f: \sum_{1} \Rightarrow \sum_{2}$, is a pair of formal systems $\left\langle\sum_{1}, \sum_{2}\right\rangle$ with language $\Lambda_{1}$ and $\Lambda_{2}$, respectively and an effective total function $f_{\Lambda}: \Lambda_{1} \rightarrow \Lambda_{2}$.

In the M\&S discipline, this definition can be interpreted as follows: $f$ in modeling, $\sum_{1}$ is a real world scenario, $\sum_{2}$ is a simulation model, $\Lambda_{1}$ is a language used to describe the scenario, and $\Lambda_{2}$ is a modeling language. One difference between the computer science and $M \& S$ fields is that $\sum_{1}$ tends to be an informal description rather than a formal system. Given this definition of abstraction, modeling is creating a mapping from a real world scenario to a model. If we consider the model information aspect, not the behavior of the model, Figure 1 is an illustration of this mapping. Here, M, the model; CS, the complete scenario; and U, 


\section{Moon and Hong}

universal information, represent the model information, the real world scenario information, and all information in the real world, respectively.

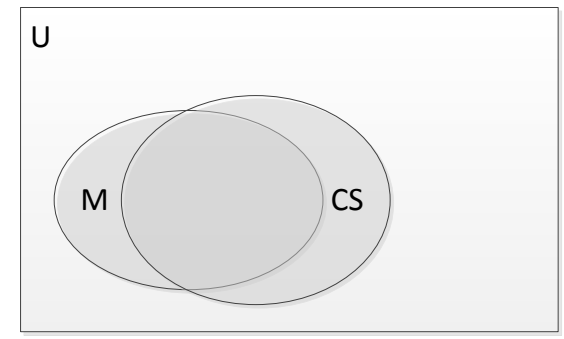

Figure 1: Illustration of abstraction with Venn diagram

To measure the level of abstraction, Benjamin et al. (1998) discusses the relationship between quantity of information and level of abstraction. They indicate that the level of abstraction of a model determines the amount of information contained in the model. The quantity of information in a model decreases with the level of abstractions. We also start our theoretic discussion from the model information perspective, similar to the concept.

Since the definition of resolution may differ from one discipline to another, M\&S articles define resolution explicitly. For example, the below is a common definition that is explicitly shown or implied in Davis (2000), Yilmaz et al. (2007), Turnitsa and Tolk (2008), and Roza (2004).

- Resolution: Resolution is a specification of the structural deviation in terms of modeling and simulation completeness; and abstraction level.

Although there is also a great amount of literature in academic and social research about defining fidelity, there still is not a clear practical and common agreed-upon definition. Each M\&S researcher provides his own definition by following related terms: accuracy and goodness. For instance, McDonald (1998) and Foster (1997) define fidelity as a synonym for accuracy, which is a measure of how well the model agrees with the real world information. Lane and Alluisi (1992) and Pace (Pace 1997; Pace 1998a; Pace 1998b) propose the definition of fidelity as the degree of correspondence between the model representation and the real world. Where the community stands, for now, is an "over-arching" definition of fidelity, which is just a consolidation of two views. Through a variety of definitions, the Fidelity Implementation Study Group (Fidelity ISG) define fidelity as follows (Gross and Freeman 1999); this definition assumes that the reproduction will be measurable or perceivable, so the measurable elements correspond to accuracy and perceivable ones to goodness.

- Fidelity: The degree to which a simulation model reproduces the state and behavior of a real system in a measurable or perceivable manner

Given the above definition, abstraction, resolution, and fidelity tend to be a selection, a degree, and a faithfulness problem respectively. In other words, abstraction makes a modeler determine what to model (Abstraction of which object). It is the process of selecting the essential aspects of a component to be represented in the model while ignoring those aspects that are irrelevant to the objective of the model. Resolution asks a modeler to how to model to a certain extent (Resolution to which level). This refers to the degree of detail used to represent aspects of the real world in a model. Fidelity indicates how faithfully a model represents a corresponding real world object or information (Fidelity to how much realism). It becomes a measure of the realism of the model. Unfortunately, we have not discovered a detailed and operationalized definition of resolution. 


\section{ABSTRACTION, RESOLUTION, AND FIDELITY OF MODEL INFORMATION}

Abstraction, resolution, and fidelity in $\mathrm{M} \& \mathrm{~S}$ can be investigated from diverse perspectives. This article particularly focuses on the model information perspective. This means that we provide a theoretic view to define and relate abstraction, resolution, and fidelity by assuming that the behavior rules of the models are most realistic for the given model information. Under this assumption, we unfold the theoretic interplay between abstraction, resolution, and fidelity by model information.

\subsection{Theoretic Articulation on Abstraction and Resolution}

According to the definition of the universal information, $U$, the model information is the set of information included in a model. Considering a simulation model, the model can be viewed as a system that takes inputs, produces outputs, and changes states, as described in Formula 1.

$\mathrm{M}(\mathrm{I} ; \mathrm{S})=0$

I: Set of Input Information, I $\subset \mathrm{U}$

S: Set of State Information, $\mathrm{S} \subset \mathrm{U}$

0 : Set of Output Information, $\mathrm{O} \subset \mathrm{U}$

For example, the DEVS formalism (B. P. Zeigler and Vahie 1993; B. Zeigler, Praehofer, and Kim 2000) is a complete representation of a discrete event model, and its model specification includes the following seven elements. Relating the formalism to the above model representation in Formula 2, I corresponds to $\mathrm{X}$ in the formalism; $\mathrm{S}$ is equal in both representations; and $\mathrm{O}$ is equal to $\mathrm{Y}$ in the formalism. The other elements, which are functions, are outside the scope of our investigation because the functions specify the model behavior with the given information. A DEVS coupled model is a composition of atomic models, and the coupled model inherits the characteristics of the atomic model by virtue of the closed under coupling property. Therefore, we can confirm that I, S, and $\mathrm{O}$ are the complete information required by the complete representation of discrete event models.

$\mathrm{AM}=<\mathrm{X}, \mathrm{Y}, \mathrm{S}, \delta_{\text {ext }}, \delta_{\text {int }}, \lambda, t a>$

$\mathrm{X}$ : Set of Input Events

Y: Set of Output Events

S: Set of Partial States

ta: Time Advance Function

$\delta_{\text {ext }}:$ Externl Transition Function

$\delta_{\text {int }}:$ Internal Transition Function

$\lambda$ : Output Function

With the complete set of information in discrete event models, we represent the model information, $\mathrm{M}$, as the union of I, S, and O. Naturally, M is the subset of the universal information U. Here, we share the symbol of the model information and the model by utilizing $\mathrm{M}$ because we limit ourselves to investigating only the information in a model, excluding its functions and its behaviors.

$M=I \cup S \cup 0$

$\mathrm{M} \subset \mathrm{U}$

Previously, we argued that the amount of real world information in a model is the level of abstraction of the model. This suggests that the size of $\mathrm{M}$, or $|\mathrm{M}|$, determines the level of abstraction from the model information perspective. This argument becomes the only axiom that we create in our theoretic development. In detail, we define the model abstraction as in Formula 4. 
$\mathrm{MA} \propto \frac{1}{|M|}$

MA: Level of Model Abstraction

The definition of model abstraction in Formula 4 enables modelers to compare the abstraction levels of two models. This comparison determines which model has more details of the real world. We operationalize this comparison by defining $>_{A}$ in Formula 5.

$M A_{M_{H A}}>M A_{M_{L A}} \Rightarrow M_{H A}>_{A} \mathrm{M}_{L A}$

$M_{H A}$ : Model with High Abstraction Level

$\mathrm{M}_{L A}$ : Model with Low Abstraction Level

When we enumerate the possible cases of two models with different abstraction, three cases are identified and illustrated in Figure 2. Figure 2 (a) suggests that the two models share no common information in the universal information, $U$, which means that the two models are abstracting completely different parts of the real world. In this case, we might compare the abstraction levels of the two models conceptually, but we cannot argue which model has more detailed information in the modeled part of the universe. Figure 2 (b) indicates that the two models focus on a common aspect of the universe, and $\mathrm{M}_{L A}$ has more information than $M_{H A}$. In Figure 2 (b), we can argue that the model with more information has more detailed information than the model with less information. Figure 2 (c) shows that the two models share a certain coverage of the universe, but they are focusing on slightly different parts overall. Although $\mathrm{M}_{L A}$ includes more information than $M_{H A}$, unlike Figure 2 (b), the two models contain different information in part. Hence, we cannot directly argue that $\mathrm{M}_{L A}$ contains more information than $M_{H A}$ regarding the modeling scope in the real world.

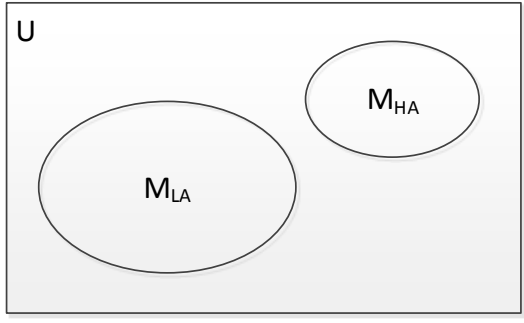

(a)

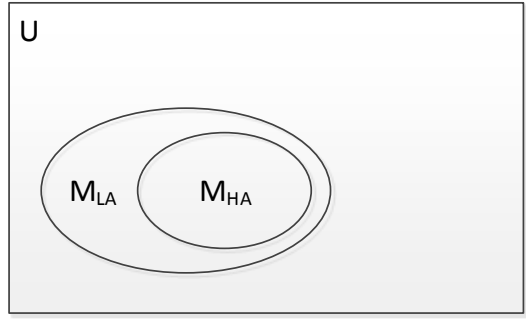

(b)

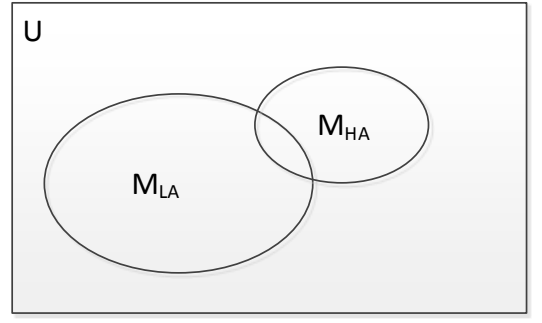

(c)

Figure 2: Three possible model information relations with different abstraction

Figure 2 (a), (b), and (c) indicate that there is a special case of abstraction comparison between the two models. Venn diagram of Figure 2 (b) suggests that the higher abstraction model is completely covered by the lower abstraction model from the model information perspective. This indicates that the information in $\mathrm{M}_{L A}$ includes the entire information in $M_{H A}$ along with additional information. Hence, this suggests that $\mathrm{M}_{L A}$ increased its resolution by adding information that is not covered by $M_{H A}$. The other cases are different from the second illustration because the models in the illustration have exclusive model information, so a direct comparison of the modeling details would not be strictly arguable. We operationalize this complete coverage of one model information to another by the operator of resolution comparison. This means that we define resolution as a concept of relation between two model abstractions. This operationalization is represented in Formula 6. This operationalization negates that the proposition in Formula 7 by the counterexamples, as shown in Figure 2 (a) and (c).

$\mathrm{M}_{L R} \subset M_{H R} \Rightarrow M_{H R}>_{R} \mathrm{M}_{L R}$

$M_{H R}$ : Model with High Resolution Level 
$\mathrm{M}_{L R}$ : Model with Low Resolution Level

$M_{H A}>_{A} \mathrm{M}_{L A} \nRightarrow M_{L A}>_{R} \mathrm{M}_{H A}$

After arguing that lower abstraction does not necessarily yield higher resolution, considering the converse of the proposition is interesting. Given the proposition in Formula 7, its converse is that the higher resolution is the lower abstraction, which is described in Formula 8. Logical proof of this converse turns out to be true, while the original proposition is false. Conversely, $\mathrm{M}_{H A}$ is a subset of $M_{L A}$ because of the definition of the resolution operator in Formula 6. A subset is always equal to or smaller than a superset, so $\mathrm{M}_{H A}$ is equal to or smaller than $M_{L A}$. This indicates that $M_{L A}$ has a lower abstraction level than $\mathrm{M}_{H A}$ according to the definition of the abstraction operator in Formula 8.

$M_{L A}>_{R} \mathrm{M}_{H A} \Rightarrow M_{H A}>_{A} \mathrm{M}_{L A}$

\subsection{Fidelity in Model Information}

The fidelity of model represents the closeness of the simulation model to the complete scenario. Since this article limits itself to the aspect of the model information, we assume that each model has most realistic representation in its model behavior. Under this assumption, the model with the highest fidelity is the model with a complete scenario in the real world. The complete scenario covers every element of information that increases the fidelity of model under the assumption of the most realistic model behavior.

Figure 3 indicates that there are two models with different model information sets and one information set that is the complete scenario, defined in Section 2.1. The models might include more information than the complete scenario, which will not affect the fidelity of the model according to the definition of the complete information of a scenario. If the model information covers only a portion of the complete scenario, which will be the case for most simulation models, the model will lack some of the information elements that would have increased the fidelity if they have been included.

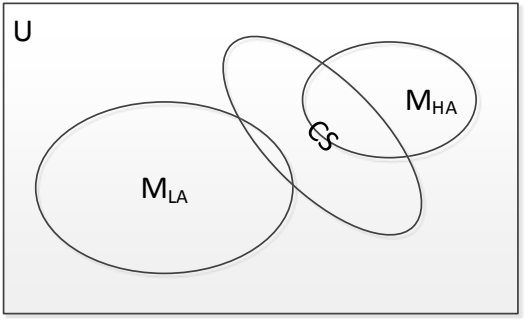

(a)

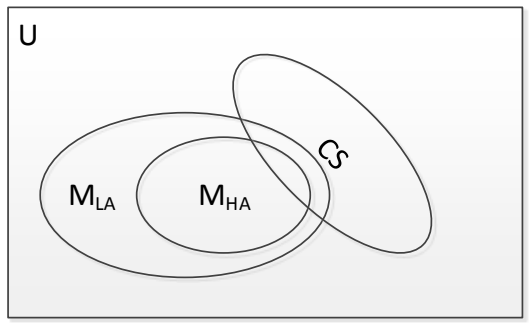

(b)

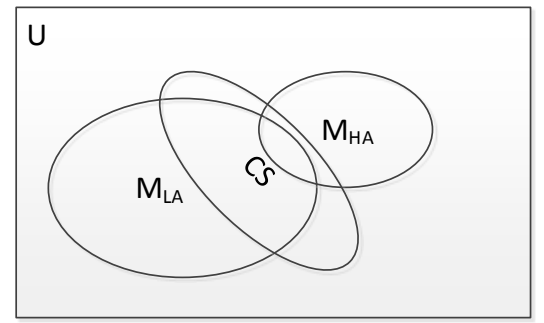

(c)

Figure 3: Three possible model information relations given a real world scenario to model

This theoretic view is applicable to the previous discussion on abstraction and resolution. Before we start applying the concept of fidelity to abstraction and resolution, we operationalize the comparison of fidelity levels between two models in Formula 9.

$\left|\mathrm{M}_{L F} \cap C S\right|<\left|\mathrm{M}_{H F} \cap C S\right| \Rightarrow M_{H F}>_{F} \mathrm{M}_{L F}$

$M_{H F}$ : Model with High Fidelity Level

$\mathrm{M}_{L F}$ : Model with Low Fidelity Level

From this operationalization, we hypothesize two propositions. The first proposition is "A lower abstraction model has higher fidelity in its model information." The second proposition is "A higher resolution model has higher fidelity in its model information." The two propositions are operationalized in Formula 10. 
1) $M_{H A}>_{A} \mathrm{M}_{L A} \nRightarrow M_{L A}>_{F} \mathrm{M}_{H A}$

2) $M_{H R}>_{R} \mathrm{M}_{L R} \Rightarrow M_{H R}>_{F} \mathrm{M}_{L R}$

The proofs of the propositions are intuitive and simple. The proof of the first proposition is done by a counterexample described in Figure 3. Figure 3 (a) shows that a lower abstraction model that is less focused on the complete scenario has lower fidelity than a higher abstraction model that is more focused on including the complete scenario. The proof of the second proposition requires a few more logical steps than the first proof, which is described in Proof 1.

$$
\begin{gathered}
M_{H R}>_{R} \mathrm{M}_{L R} \\
\Rightarrow \mathrm{M}_{L R} \subset M_{H R} \\
\Rightarrow \mathrm{M}_{L R} \cap C S \subset M_{H R} \cap C S \\
\Rightarrow\left|\mathrm{M}_{L R} \cap C S\right|<\left|M_{H R} \cap C S\right| \\
\Rightarrow M_{H R}>_{F} \mathrm{M}_{L R} \\
\therefore M_{H R}>_{R} \mathrm{M}_{L R} \Rightarrow M_{H R}>_{F} \mathrm{M}_{L R}
\end{gathered}
$$

Proof 1: Proof on proposition between resolution and fidelity relations

\subsection{Theoretic Relation of Abstraction, Resolution, and Fidelity}

This subsection summarizes the theoretic relations of abstraction, relation, and fidelity, which are identified in this article through definitions and proofs. Figure 4 is the illustrative version of the summary. According to the definitions, resolution is a special case of abstraction. Regardless of the intersection between two model information sets, lower abstraction means more model information than a higher abstraction. Meanwhile, higher resolution means that the model information is a superset of the model information in a lower resolution model. This definition difference results in two logical statements. First, a higher resolution model is a lower abstraction model. Second, a lower abstraction model is not necessarily a higher resolution model.

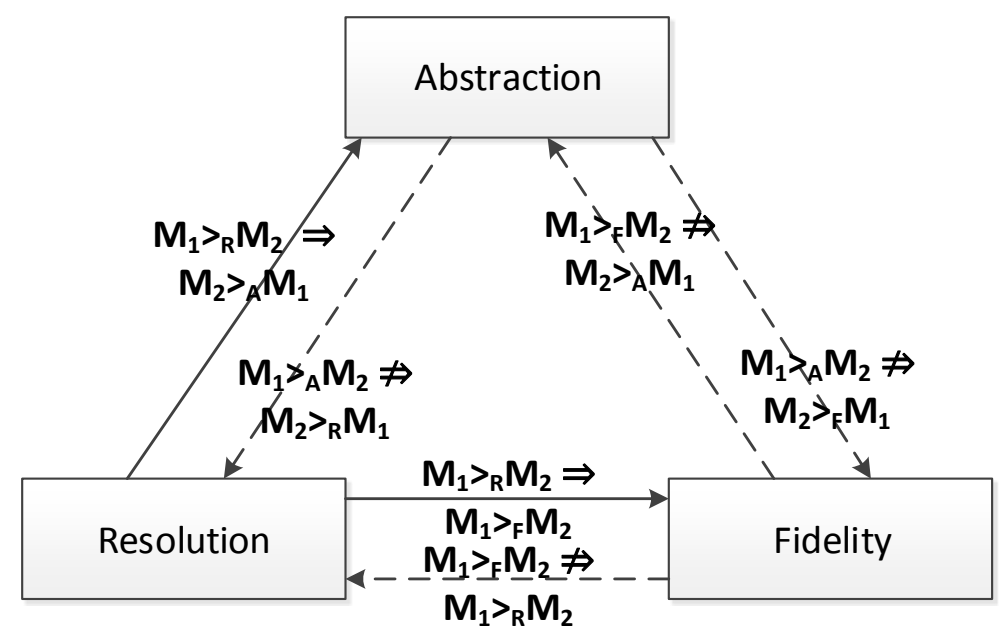

Figure 4: Illustration of theoretic relation of abstraction, resolution, and fidelity

When the concept of fidelity joins this theoretic relation, this discussion involves a real world scenario of interest and its complete information to model. A model with higher fidelity should cover more model information belonging to the complete information set of the scenario. Then, this article proved two logical statements. First, a lower abstraction model does not necessarily have high fidelity. Second, a higher resolution model should have high fidelity. These statements are true only when we assume the most realistic 


\section{Moon and Hong}

model behavior given the set of model information. If this assumption is not guaranteed, the theoretic relations should be evaluated accordingly.

\section{IMPLICATIONS AND LIMITATIONS}

This work aims to deliver more concrete ground for M\&S researchers to discuss abstraction, resolution, and fidelity. These concepts are the backbones of the M\&S discipline, but many M\&S researchers use the terms and apply the concepts causally. However, we argue that these concepts need to be used more consciously because the model users fundamentally rely on these concepts to understand simulation models. To support the articulation of abstraction, resolution, and fidelity, we utilize the set theory to represent and investigate the meanings of and relations among these concepts. In particular, we focus on the model information aspect of these concepts under the assumption that models provide the most realistic behavior with available information.

This work is preliminary and requires further development. First, this work should be developed as a joint investigation within the M\&S community. The presented theoretic development should be carefully examined by active members of the research community, and consensus on the work is imperative for wide acceptance. Second, the presented theoretic contribution should conform to the existing M\&S theories and the theoretic framework. This work should position itself as a supporting framework, not an obstruction to the current practices. One distinct characteristics of $\mathrm{M} \& \mathrm{~S}$ is its practical approach to real world problems. Theoretic contributions should be developed in conjunction with the practical approaches. For example, the DEVS formalism, one of the theoretic works in M\&S, is better recognized and utilized when the formalism is used to solve real world problems, such as defense modeling and simulation. Third, this work should examine the effect of model behavior that is beyond the scope of model information. Two models can be completely different from each other even if they use the same information.

\section{ACKNOWLEDGMENTS}

This work was supported by the Public welfare \& safety research program through the National Research Foundation of Korea (NRF) (2012-0029881). This work was also supported by Defense Acquisition Program Administration and Agency for Defense Development under the contract UD110006MD, Korea

\section{REFERENCES}

Benjamin, P., M. Erraguntla, D. Delen, and R. Mayer. 1998. "Simulation Modeling at Multiple Levels of Abstraction." In Proceedings of the 1998 Winter Simulation Conference, ed. D. J. Medeiros, E. F. Watson, J. S. Carson, and M. S. Manivannan, 391-398. Piscataway, New Jersey: Institute of Electrical and Electronics Engineers, Inc.

Davis, P. K. 2000. "Exploratory Analysis Enabled By Multiresolution, Multiperspective Modeling." In Proceedings of the 2000 Winter Simulation Conference, ed. J. A. Joines, R. R. Barton, K. Kang, and P. A. Fishwick, 293-302. Orlando, FL: Piscataway, New Jersey: Institute of Electrical and Electronics Engineers, Inc.

Davis, P. K., and J. H. Bigelow. 1998. Experiments in Multiresolution Modeling (MRM). 1st ed. Santa Monica, CA: RAND Corp.

Davis, P. K., and A. Tolk. 2007. "Observations of New Developments in Composability and MultiResolution Modeling." In Proceedings of the 2007 Winter Simulation Conference, ed. S. G. Henderson, B. Biller, M.-H. Hsieh, H. Shortle, J. D. Tew, and R. R. Barton, 859-870. Washington, D.C.: Piscataway, New Jersey: Institute of Electrical and Electronics Engineers, Inc.

Foster, L. 1997. "Fidelity in Modeling and Simulation." In Proceeding of the 1997 Spring Simulation Interoperability Workshop, 97S-SIW-073.

Giunehiglia, F., and T. Walsh. 1992. "A Theory of Abstraction." Artificial Intelligence 57: 323-389.

Gross, D. C., and R. H. Freeman. 1997. "Measuring Fidelity Differentials in HLA Simulations." In The 1997 Fall Simulation Interoperability Workshop, 97F-SIW-125. 
Gross. 1999. "Report from the Fidelity Implementation Study Group." In The 1999 Spring Simulation Interoperability Workshop, 99S-SIW-167.

Gross, D. C., R. H. Freeman, and V. T. William. 1998. "A Case Study in Fidelity Differential Measurements." In Proceeding of the 1998 Spring Simulation Interoperability Workshop, 98S-SIW185.

Lane, N. E., and E. A. Alluisi. 1992. Fidelity and Validity in Distributed Interactive Simulations: Questions and Answers. Alexandria, VA.

McDonald, B. 1998. "A Proposed Process for Defining Required Fidelity of Simulations." In The 1998 Spring Simulation Interoperability Workshop, 98S-SIW-234.

Mitchell, B., and A. Yilmaz. 2008. "Symbiotic Adaptive Multisimulation: An Autonomic Simulation Framework for Real-Time Decision Support Under Uncertainty." ACM Transactions on Modeling and Computer Simulation2 19 (2): 2:1-2:31.

Pace, D. K. 1997. "Fidelity Considerations for RDE Distributed Simulation." In The 1997 Fall Simulation Interoperability Workshop, 97F-SIW-004.

Pace. 1998a. "Dimensions and Attributes of Simulation Fidelity, Draft Version." In The 1998 Fall Simulation Interoperability Workshop, 98F-SIW-017.

Pace. 1998b. "Synopsis of Fidelity Ideas and Issues." In The 1998 Spring Simulation Interoperability Workshop, 98S-SIW-071.

Roza, Z. C. 2004. "Simulation Fidelity Theory and Practice: A Unified Approach to Defining, Specifying and Measuring the Realism of Simulations". Doctoral Thesis, Dept. of Aerospace Engineering, Delft University of Technology.

Turnitsa, C., and A. Tolk. 2008. "Knowledge Representation and the Dimensions of a Multi-Model Relationship." In Proceedings of the 2008 Winter Simulation Conference, ed. S. J. Mason, R. R. Hill, L. Monch, O. Rose, T. Jefferson, and J. W. Fowler, 1148-1156. Miami, FL: Piscataway, New Jersey: Institute of Electrical and Electronics Engineers, Inc.

Yilmaz, L., A. Lim, S. Bowen, and T. Oren. 2007. "Requirements and Design Principles for Multisimulation with Multiresolution, Multistage Multimodels." In Proceedings of the 2007 Winter Simulation Conference, ed. S. G. Henderson, B. Biller, M.-H. Hsieh, H. Shortle, J. D. Tew, and R. R. Barton, 823-832. Washington, D.C.: Piscataway, New Jersey: Institute of Electrical and Electronics Engineers, Inc.

Zeigler, B P, and S Vahie. 1993. "DEVS Formalism and Methodology: Unity of Conception/diversity of Application." In Proceedings of the 25th Conference on Winter Simulation, ed. G. W. Evans, M. Mollaghasemi, E. C. Russell, and W. E. Biles, 573-579. Piscataway, New Jersey: Institute of Electrical and Electronics Engineers, Inc.

Zeigler, BP, Herbert Praehofer, and Tag Gon Kim. 2000. Theory of Modeling and Simulation: Integrating Discrete Event and Continuous Complex Dynamic Systems. 2nd Editio. New York: Academic Press.

\section{AUTHOR BIOGRAPHIES}

IL-CHUL MOON is an assistant professor at Dept. of Industrial and Systems Engineering, KAIST. His theoretic research focuses on the overlapping area of computer science, management, sociology and operations research. His practical research includes military command and control analysis, counter-terrorism analysis, intelligence analysis, and disaster management. His email address is icmoon@kaist.ac.kr.

JUNG HEE HONG is a post-doctoral researcher at Dept. of Industrial and Systems Engineering, KAIST. Her theoretic research focuses on systems engineering. Particularly, she has interest in discrete event systems modeling and simulation, simulation-based optimization, and distributed simulation. Her practical research includes High Level Architecture (HLA) and large scale system simulation such as defense system. Her email address is jeonghee.hong@gmail.com 